\title{
Association of CETP Taq1B and -629 C > A polymorphisms with coronary artery disease and lipid levels in the multi-ethnic Singaporean population
}

Yongjian $\mathrm{Lu}^{2+}{ }^{+}$, Naeimeh Tayebi ${ }^{1 \dagger}$, Hongzhe $\mathrm{Li}^{1}$, Nilmani Saha ${ }^{1}$, Hongyuan Yang ${ }^{2,3}$ and Chew-Kiat Heng ${ }^{1 *}$

\begin{abstract}
Background: Hyperlipidaemia is a major risk factor for coronary artery disease (CAD) and cholesteryl ester transfer protein (CETP) gene polymorphisms are known to be associated with lipid profiles.

Methods: In this study, we investigated the association of two polymorphisms in the CETP, Taq1B (rs708272) and -629C > A (rs1800775), with CAD and lipid levels HDL-C in 662 CAD + cases and 927 controls from the Singapore population comprising Chinese, Malays and Indians.

Results: TaqB2 frequency was significantly lowest in the Malays (0.43) followed by Chinese (0.47) and highest in the Indians (0.56) in the controls. The B2 allele frequency was significantly lower in the Chinese CAD + cases compared to the controls $(p=0.002)$. The absence of the B2 allele was associated with CAD with an OR $2.0(95 \% \mathrm{Cl} 1.2$ to 3.4) after adjustment for the confounding effects of age, smoking, BMI, gender, hypertension, dyslipidemia and diabetes mellitus. The B2 allele was significantly associated with higher plasma HDL-C levels in the Chinese men after adjusting for confounders. Associations with plasma apoA1 levels were significant only in the Chinese men for Taq1B and $-629 C>A$. In addition, the Taq1B polymorphism was only associated with plasma Apo B and $L p(a)$ in the Malay men. Significant associations were only found in non-smoking subjects with $B M l<50^{\text {th }}$ percentile. In this study, the LD coefficients between the Taq1B and $-629 C>$ A polymorphisms seemed to be weak.

Conclusion: The absence the Taq1B2 allele was associated with CAD in the Chinese population only and the minor allele of the Taq1B polymorphism of the CETP gene was significantly associated with higher plasma HDL-C levels in Chinese men.
\end{abstract}

Keywords: Cholesteryl ester transfer protein, -629C > A polymorphism, TaqB1 polymorphism, HDL-cholesterol, Coronary artery disease

\section{Introduction}

The inverse relationship between plasma high density lipoprotein-cholesterol (HDL-C) and the incidence of coronary artery disease (CAD) is well established [1,2]. HDL is thus believed to be a protective factor against CAD. The removal of cholesterol from tissues and its delivery to the liver for excretion constitute the process of reverse transport $[3,4]$. One of the important components

\footnotetext{
* Correspondence: paehck@nus.edu.sg

${ }^{\dagger}$ Equal contributors

'Department of Paediatrics, Yong Loo Lin School of Medicine,

National University of Singapore, Singapore, Singapore

Full list of author information is available at the end of the article
}

of this process is the cholesteryl ester transfer protein (CETP). CETP facilitates the transfer of cholesteryl ester from HDL to triglyceride-rich lipoproteins in exchange for triglycerides (TG) $[5,6]$. Many CETP DNA polymorphisms that may alter the function of CETP have been reported [7-9]. However, the association of these polymorphisms with phenotypes such as lipid levels and the CAD remain inconsistent [10-14].

Among the several CETP polymorphisms reported, some are found to be associated with plasma levels of HDL-C. One of the most widely studied polymorphic sites on the CETP gene is the Taq1B site $[15,16]$. This is a polymorphism at nucleotide 277 in intron $1(277 \mathrm{C}>\mathrm{T}$,

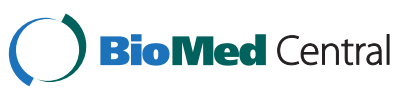


rs708272). However, the $B 2$ allele of this polymorphism has been shown to be associated with elevated plasma HDL-C levels in numerous studies [17-21]. It has been reported that the Taq1B site is in linkage disequilibrium (LD) with another polymorphic site $(-629 C>A, \mathrm{rs} 1800775)$ in the CETP promoter region [22,23] and the latter could explain the association between Taq1B and HDL-C levels since it was shown to be a functional base substitution. However, a subsequent study in the Icelandic population reported that the $-629 C>A$ polymorphism does not explain the association of Taq1B polymorphism with risk of myocardial infarction although both are associated with HDL-C levels [24].

The effects of CETP polymorphisms have been reported in some of the Asian populations such as Sri Lankans [25], Koreans [26],Taiwanese [27,28] Chinese [29], Indians [30,31] and Turkish [32].The multi-ethnic Singaporean population is also studied for the Taq1B and $-629 C>A$ polymorphisms at the CETP locus for their association with lipid levels [33]. However, the study was conducted in the general population and hence the association of the polymorphisms with CAD could not be determined. On the other hand it is noted that the mortality rate of CAD in the ethnic Asian Indians is more than three times of the Chinese [34]. The impact of the CETP genetic variants on complex outcomes such as CAD is also warranted by Thompson et al. [8]. As far as lipid profile is concerned the plasma HDL-C level is shown to be one of the most discriminative factors for CAD in Singapore population [35].

Singapore offers a good context for studying the genetic effects on CAD and lipid levels as three major diverse ethnic groups are residing in this small island state. We report here the allele frequencies and the association of the CETP Taq1B and $-629 C>A$ polymorphisms with CAD and lipid levels in the Chinese, Malays and Asian Indians residing in Singapore.

\section{Materials and methods \\ Ethics}

This study was approved by the institutional ethics committee of National University of Singapore (NUS) IRB and all subjects gave their informed consent for their participation in the study.

\section{Study design}

This was a case-control study. The cases were the angiographically confirmed CAD patients while the controls were selected from the general population who attended routine medical examination at a health screening centre as required by their employers.

\section{Study subjects}

A total of 662 angiographically confirmed CAD patients $(\mathrm{CAD}+)$ who were admitted consecutively for coronary artery by-pass graft at Singapore's National Heart Centre between 1995 and 2002 were included as cases in this study. All patients had more than $50 \%$ stenosis in at least one of the major coronary arteries as diagnosed by angiography.

Separately, 927 consecutive individuals from the general population, who attended routine medical examination at a community screening centre, without the history of heart disorders and ECG abnormalities were recruited as controls.

\section{Sample collection and biochemical tests}

The blood samples from the CAD + cases were collected before their by-pass operation for routine examination including lipid levels. The blood samples from the controls were collected in tubes containing EDTA as anti-coagulant after an overnight fast of at least 10 hours as a part of routine examinations and tests. The plasma was separated by centrifugation and aliquot portions were stored at $-20^{\circ} \mathrm{C}$ until use. Measurement of plasma lipids and DNA extraction were carried out as previously described [36].

\section{Genotyping}

The Taq1B polymorphism was genotyped by restriction fragment length polymorphism (RFLP) assay. A 535 bp fragment in the intron 1 of the CETP gene was amplified by using the primers $5^{\prime}$ CACTAGCCCAGAGAGA GGAGTGCC3' and 5' CTGAGCCCAGCCGCACACT AAC3'. Amplification was carried out in a volume of 20 $\mu \mathrm{l}$ containing $1-10 \mu \mathrm{g}$ of genomic DNA, $20 \mathrm{pmol}$ of each primer, $0.5 \mathrm{mM}$ of each dNTP, reaction buffer, $2 \%$ DMSO and 1.0 U of Taq DNA polymerase. Amplified products were digested overnight with $8 \mu \mathrm{l}$ of Taq1 at $65{ }^{0} \mathrm{C}$. The resulting fragments were 174 and $361 \mathrm{bp}$ for $B 1$ allele and 535 bp for B2 allele.

Allele specific amplification was used to identify the $629 C>A$ promoter polymorphism. The common sense primer was GGCAGCTTTGGTATTGGAG. The specific anti-sense primers for the $A$ and $C$ allele were GATAT GCATAAAATAACTCTGAGG and GATATGCATAAAA TAACTCTGAGT respectively. We found that creating a deliberate mismatch at the $-627 \mathrm{C}$ nucleotide by changing it to $A$ in both primers could yield better allele discrimination. The amplicons were visualized by electrophoresis on $2.5 \%$ agarose gel. The specific amplified fragment was 398 bp in length. Another pair of primers from ABCA1 gene was introduced into the system as an amplification control: sense, CTTCACTCCCATATTTCAGAACTTG and anti-sense, ATCTCCATTAAAGCATCCTACAGC. The control product was $276 \mathrm{bp}$ long.

\section{Statistical analysis}

The majority of statistical analysis was performed using SPSS Version 19. The chi-squared $\left(x^{2}\right)$ analysis was used 
to test for departure from Hardy-Weinberg equilibrium (HWE) and for differences in allele frequencies between groups. Analysis of covariance (ANCOVA) was performed to determine the effects of the CETP gene polymorphisms on the various lipid traits using significant confounding factors such as age, BMI and cigarette smoking as covariates. The significance of the sample variance was tested by $F$ and $P$ values while percentage of explained variance $\left(R^{2} \times 100\right)$ was calculated from the sum of squares. Due to their skewed distribution, the raw data for TG and $\mathrm{Lp}(\mathrm{a})$ were normalized by natural logarithm transformation prior to analysis. The LD between the Taq1B and $-629 C>A$ sites was indicated by $r^{2}$. Statistical significance was taken at $\alpha=0.05$.

In the combined samples, the association analysis was performed between plasma HDL-C levels and CETP gene polymorphisms using linear model and was also assessed for the homogeneity using Cochrane's $Q$ statistic and $\mathrm{I}^{2}$ heterogeneity index in meta-analysis in PLINK.

Wherever appropriate, the analyses were performed separately for men and women given the differences in lipid levels that exist in these groups. The association between CETP polymorphism and lipid profile was evaluated only in the control group because the lipid levels in the CAD + cases may be influenced by the lipid lowering agents that some of these patients had received following the diagnosis of CAD.

\section{Results}

\section{Subject characteristics}

The subject characteristics of the study population for three ethnic populations for CAD + and controls are given in Table 1.

Significant differences between CAD + cases and controls were observed for most components of the lipid profiles. Among these, TC, LDL-C, TG and apolipoprotein (apo) B were significantly lower in the CAD + cases as against the controls of all ethnic groups. Proportion of subjects with Hypertension and diabetes was significantly higher in CAD + cases as compared to control. However, higher proportion of subjects was dislipedemic in controls as compare to cases. These were expected as most CAD + cases were on lipid-lowering drugs. Plasma levels of $\mathrm{Lp}(\mathrm{a})$, which is very resistant to modification, was significantly higher in the CAD + cases as against the controls in all ethnic groups. As the mean age of the $\mathrm{CAD}+$ cases was significantly higher than the controls, we carried out logistic regression to determine if age was significantly related to the allele frequencies of both polymorphisms and found that it was not. We were

Table 1 Demographics of the study subjects in three ethnic populations

\begin{tabular}{|c|c|c|c|c|c|c|c|c|c|}
\hline \multirow[b]{2}{*}{ Variables } & \multicolumn{3}{|c|}{ Chinese } & \multicolumn{3}{|c|}{ Malays } & \multicolumn{3}{|c|}{ Indians } \\
\hline & $\begin{array}{l}\text { Control } \\
n=383\end{array}$ & $\begin{array}{c}\text { CAD+ } \\
n=442\end{array}$ & $P$ & $\begin{array}{l}\text { Control } \\
n=155\end{array}$ & $\begin{array}{c}\text { CAD+ } \\
n=110\end{array}$ & $P$ & $\begin{array}{l}\text { Control } \\
n=389\end{array}$ & $\begin{array}{c}\text { CAD+ } \\
n=110\end{array}$ & $P$ \\
\hline Age (years) & $42.74 \pm 14.2$ & $59.34 \pm 8.9$ & $<0.0005$ & $40.66 \pm 9.24$ & $59.08 \pm 9.18$ & $<0.0005$ & $42.43 \pm 14.08$ & $60.35 \pm 10.25$ & $<0.0005$ \\
\hline BMI $\left(\mathrm{kg} / \mathrm{m}^{2}\right)$ & $23 \pm 3.61$ & $24.24 \pm 3.67$ & ns & $25.17 \pm 4.06$ & $26.14 \pm 3.62$ & ns & $24.77 \pm 4.8$ & $24.9 \pm 3.2$ & ns \\
\hline Smokers* (\%) & 17.4 & 52.9 & $<0.0005$ & 52.7 & 49 & ns & 13.6 & 43.8 & $<0.0005$ \\
\hline \multicolumn{10}{|l|}{ Sex (\%) } \\
\hline Female & 46.1 & 21.9 & $<0.0005$ & 8.7 & 23.9 & 0.001 & 37.8 & 16.5 & $<0.0005$ \\
\hline Male & 53.9 & 78.1 & & 91.3 & 76.1 & & 62.2 & 83.5 & \\
\hline Dyslipidemia & $46.3 \%$ & $32.6 \%$ & $<0.0005$ & $56.4 \%$ & $27.5 \%$ & $<0.0005$ & $63.4 \%$ & $21.1 \%$ & $<0.0005$ \\
\hline Hypertension & $8.2 \%$ & $69.1 \%$ & & $4.1 \%$ & $72.7 \%$ & & $9.8 \%$ & $61.8 \%$ & \\
\hline Diabetes Mellitus & $2.7 \%$ & $43.3 \%$ & & $3.4 \%$ & $62.6 \%$ & & $6.4 \%$ & $61.8 \%$ & \\
\hline $\mathrm{TC}(\mathrm{mM})$ & $5.81 \pm 1.19$ & $4.57 \pm 1.14$ & $<0.0005$ & $5.85 \pm 1.22$ & $4.6 \pm 1.13$ & $<0.0005$ & $5.61 \pm 1.22$ & $4.25 \pm 1.99$ & $<0.0005$ \\
\hline $\mathrm{HDL}-\mathrm{C}(\mathrm{mM})$ & $1.4 \pm 0.4$ & $0.97 \pm 0.26$ & $<0.0005$ & $1.19 \pm 0.27$ & $0.91 \pm 0.27$ & $<0.0005$ & $1.11 \pm 0.32$ & $0.88 \pm 0.23$ & $<0.0005$ \\
\hline LDL-C (mM) & $3.57 \pm 1.17$ & $2.78 \pm 0.99$ & $<0.0005$ & $3.7 \pm 1.23$ & $2.89 \pm 1.03$ & $<0.0005$ & $3.62 \pm 1.15$ & $2.58 \pm 0.86$ & $<0.0005$ \\
\hline $\mathrm{TG}(\mathrm{mM})$ & $1.84 \pm 1.9$ & $1.37 \pm 0.66$ & 0.008 & $2.09 \pm 1.31$ & $1.51 \pm 0.68$ & 0.014 & $1.93 \pm 1.45$ & $1.29 \pm 0.63$ & $<0.0005$ \\
\hline ApoA1 (mg/dl) & $144.4 \pm 22.04$ & $119.47 \pm 20.5$ & $<0.0005$ & $129.29 \pm 18.5$ & $115.29 \pm 20.8$ & $<0.0005$ & $135.85 \pm 25.11$ & $110.01 \pm 19.8$ & $<0.0005$ \\
\hline ApoB (mg/dl) & $106.2 \pm 31.4$ & $89.78 \pm 22.6$ & $<0.0005$ & $121.36 \pm 29$ & $103.64 \pm 28$ & $<0.0005$ & $122.87 \pm 33.04$ & $102.3 \pm 26.5$ & $<0.0005$ \\
\hline Lp(a) (mg/dl) & $16.90 \pm 22.67$ & $24.33 \pm 27.38$ & 0.007 & $14.66 \pm 15.16$ & $19.5 \pm 16.28$ & 0.032 & $21.9 \pm 21.17$ & $26.89 \pm 11.4$ & 0.038 \\
\hline
\end{tabular}

Age, BMI and plasma lipid profiles are presented in mean \pm SD. Continues and categorical data were tested using independent sample $t$-test and Chi-square test respectively.

TC, total cholesterol; HDL-C, high-density lipoprotein cholesterol; LDL-C, low-density lipoprotein cholesterol; TG, triglyceride; BMI, body mass index; Apo, Apolipoprotein; Lp(a), lipoprotein(a).

*Smokers and ex-smokers form one category while non-smokers constitute another. 
therefore confident that any significant differences in allele frequencies between CAD + cases and controls were not due to the confounding effects of age.

\section{Frequency distribution of the polymorphisms}

Figure 1 shows the genotype and allele distributions of the two polymorphisms in the controls and CAD + cases, among the three ethnic groups in Singapore.
Based on frequencies in the controls, the $B 2$ allele frequency was lowest in the Malays $(0.43)$ followed by Chinese (0.47) and highest in the Indians (0.56). The B2 frequency was significantly lower in Malay controls as compared to the Indians $(\mathrm{p}=0.01)$ and Chinese $(\mathrm{p}=0.005)$ controls. In all ethnic groups, $B 1$ was the major allele.

The $-629 C$ allele was predominant in Chinese (0.52) and Malays (0.60) controls, but not in Indians (0.30).

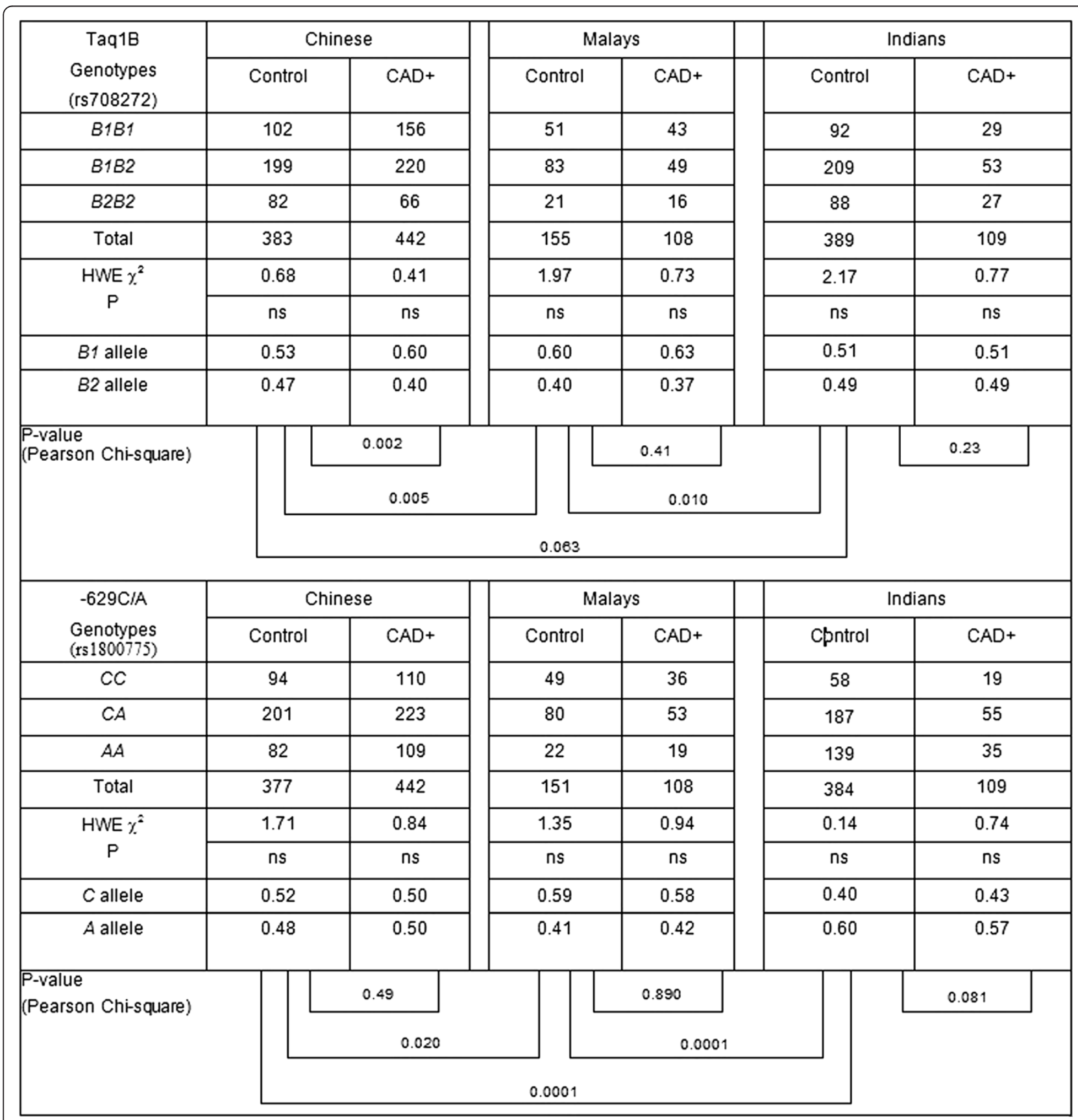

Figure 1 Genotype and allele frequencies of the TaqIB and -629C/A promoter polymorphisms in the three ethnic populations for CAD+ cases and controls. HWE $x^{2}: x^{2}$-test for departure from Hardy Weinberg equilibrium. Pearson Chi-Square is the test statistic for allele frequency comparison between controls and CAD+ cases. P-values were obtained after adjustment for gender, age, smoking status, body mass index, hypertension, dyslipidemia and diabetes mellitus. 
Allele frequencies significantly varied between the controls of all ethnic groups. The genotype distributions of both polymorphisms in all three ethnic groups were consistent with Hardy Weinberg expectations.

\section{Case-control differences in allele frequencies}

Apart from the highly significant lower B2 allele in the Chinese CAD + cases compared to the controls $(\mathrm{p}=0.002)$, the allele frequencies for both the promoter and Taq1B polymorphisms are not significantly different between CAD + cases and controls in all the ethnic groups.

The odds ratio for CAD associated with the absence of B2 allele is 2.0 (95\% CI 1.2 to 3.4) after adjustments were made for the confounding effects of age, cigarette smoking, BMI, gender, hypertension, dyslipidemia and diabetes mellitus by logistic regression. The absence of $B 2$ allele is hence independently associated with CAD in the Chinese but not for the Malays and Indians. There is no significant association of the $-629 C>A$ polymorphism with CAD.

\section{LD between Taq1B and-629C $>A$ sites}

The LD coefficients between the Taq1B and $-629 C>A$ polymorphisms are presented in Table 2 . It is interesting to note that LD is stronger in control than in cases for the Chinese (0.38 vs. 0.10$)$ and Malays (0.38 vs. 0.07$)$, but not for the Indians $(0.11$ vs. 0.12$)$. With the maximum $\mathrm{r}^{2}$ value in controls being 0.388 , considering the cut off of 0.80 [37], there seems to be a weak LD between the two polymorphism studied.

\section{Impact of CETP polymorphisms on plasma lipid profile}

The association of the Taq1B and $-629 C>A$ polymorphisms with lipid levels in our population show ethnic and gender-specificity (Tables 3 and 4). All analyses were carried out with age, BMI, smoking status, hypertension and diabetes mellitus as covariates. Consistent association of the minor allele $B 2$ with higher plasma HDL-C levels in the Chinese men was observed in the Taq1B polymorphism studied. Plasma HDL-C level was in the order $B 1 B 1<B 1 B 2<B 2 B 2(\mathrm{p}=0.004)$. Associations with plasma apoA1 levels were significant only in the Chinese

Table 2 Linkage disequilibrium coefficients between the Taq1B and $-629 C / A$ sites in the three ethnic populations for CAD+ cases and controls

\begin{tabular}{lll}
\hline & & $r^{2}$ \\
\hline \multirow{2}{*}{ Controls } & Chinese & 0.380 \\
& Malays & 0.388 \\
& Indians & 0.113 \\
CAD+ & Chinese & 0.101 \\
& Malays & 0.074 \\
& Indians & 0.128 \\
\hline
\end{tabular}

men for $\operatorname{Taq1B}(\mathrm{p}<0.001)$ and $-629 C>A \quad(\mathrm{p}=0.029)$. The other lipids that are significantly associated with the polymorphisms are shown in Tables 3 and 4 .

Since there are two polymorphisms on the same CETP gene, we examined the combined effects of both polymorphisms on plasma lipid levels using their composite genotypes. All 9 possible combinations of genotypes are present in our population. However, two composite genotypes, $C C / B 2 B 2$ and $A A / B 1 B 1$, were excluded from the analysis because there was only one subject in the former and two subjects in the latter although their levels are still shown in Figure 2. Significant effects of the composite genotypes on HDL-C and apoA1 in the Chinese men are observed. ANCOVA results showed that these are highest when both polymorphic sites are homozygous for the minor alleles (Figure 2).

We also carried out two-way ANCOVA to assess the effects of the two polymorphisms on HDL-C and apoA1 levels in the control group of Chinese men. When the Taq1B polymorphism was included in the two-way ANCOVA model with adjustment for the confounding effects of age, BMI, smoking, hypertension and diabetes mellitus, the $-629 C>A$ promoter site showed no significant association with HDL-C and apoA1 ( $\mathrm{p}=0.455$ and 0.812 , respectively). However, in the presence of the promoter genotypes, the Taq1B polymorphism remained significantly associated with HDL-C and apoA-1 ( $\mathrm{p}=$ 0.039 and 0.009 respectively) indicating that the effects of the Taq1B polymorphism are independent and not due to its weak LD with the promoter polymorphism.

Moreover, we performed a fixed effect meta-analysis across all three populations $\left(\mathrm{Q}>0.05\right.$ and $\left.\mathrm{I}^{2}=0.00\right)$. The Taq1B polymorphism showed highly significant association with plasma HDL-C in the combined population $(\mathrm{p}=5.502 \mathrm{e}-06)$. However, $-629 \mathrm{C}>\mathrm{A}$ had no association with HDL-C ( $\mathrm{p}=0.0991)$.

\section{Gene-environmental interaction}

Since BMI was found to be a significant confounding factor of HDL-C and apoA1 levels, we examined the interaction of CETP genotypes with BMI. Figure 3A shows that the protective effect of the $B 2$ allele was only apparent for Chinese men subjects having BMI lower than the $50^{\text {th }}$ percentile, beyond which, HDL-C levels were no longer significantly different between genotypes. This was confirmed by carrying out ANCOVA separately for subjects below and above the $50^{\text {th }}$ percentile. The Taq1B polymorphism effect was only significant $(\mathrm{p}=0.007)$ for the lower 2 quartiles but not the upper two $(\mathrm{p}=0.302)$. The $-629 C>A$ promoter polymorphism followed a similar trend in the Chinese men (Figure $3 \mathrm{~B}$ ).

Another environmental factor which interacted with the Taq1B genotypes is cigarette smoking. We found significant protective effect of the $B 2$ allele only in non-smoking 
Table 3 Genotypic lipid levels (Mean \pm SD) of the Taq1B polymorphism in the three ethnic populations for controls

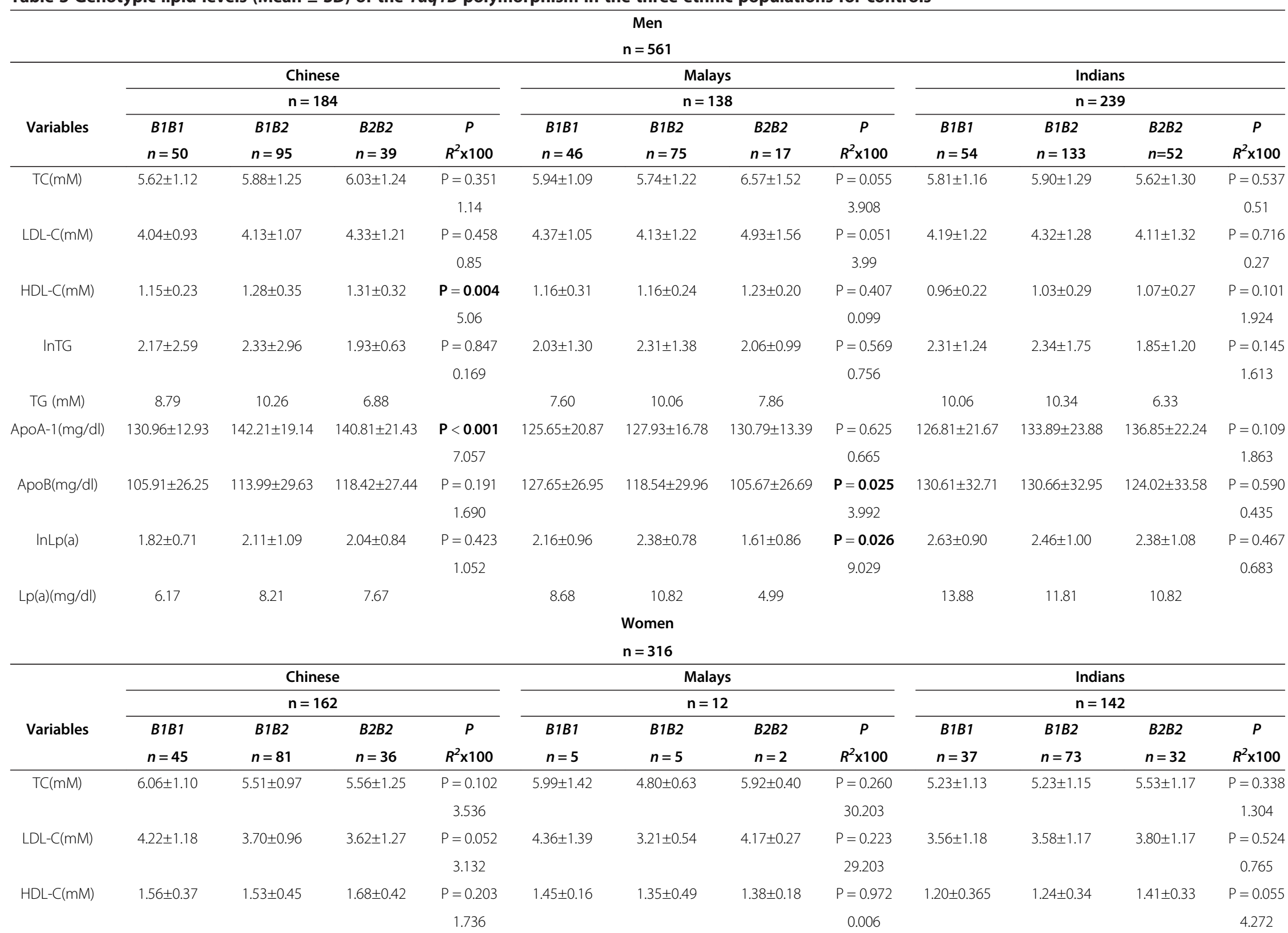


Table 3 Genotypic lipid levels (Mean \pm SD) of the Taq1B polymorphism in the three ethnic populations for controls (Continued)

\begin{tabular}{|c|c|c|c|c|c|c|c|c|c|c|c|c|}
\hline \multirow[t]{2}{*}{$\operatorname{lnTG}$} & $1.41 \pm 0.74$ & $1.41 \pm 0.84$ & $1.25 \pm 0.75$ & $P=0.733$ & $0.89 \pm 0.33$ & $1.24 \pm 1.01$ & $1.86 \pm 1.53$ & $P=0.203$ & $1.54 \pm 1.13$ & $1.55 \pm 1.32$ & $1.20 \pm 0.62$ & $P=0.291$ \\
\hline & & & & 0.326 & & & & 20.65 & & & & 1.681 \\
\hline $\mathrm{TG}(\mathrm{mM})$ & 4.11 & 4.11 & 3.50 & & 2.43 & 3.44 & 6.42 & & 4.67 & 4.71 & 3.31 & \\
\hline \multirow[t]{2}{*}{ ApoA-1 (mg/dl) } & $147.24 \pm 20.68$ & $150.59 \pm 25.73$ & $152.88 \pm 21.93$ & $P=0.561$ & $144.13 \pm 11.75$ & $146.33 \pm 23.79$ & $149.33 \pm 8.50$ & $P=0.541$ & $136.50 \pm 23.30$ & $139.35 \pm 26.63$ & $150.97 \pm 28.83$ & $P=0.07$ \\
\hline & & & & 0.709 & & & & 5.537 & & & & 3.773 \\
\hline \multirow[t]{2}{*}{ ApoB(mg/dl) } & $96.98 \pm 25.75$ & $89.72 \pm 21.93$ & $88.75 \pm 32.74$ & $P=0.476$ & $99.80 \pm 26.75$ & $95.40 \pm 10.45$ & $109.00 \pm 2.83$ & $P=0.459$ & $113.78 \pm 32.03$ & $110.51 \pm 31.06$ & $117.35 \pm 33.01$ & $P=0.596$ \\
\hline & & & & 0.900 & & & & 14.32 & & & & 0.57 \\
\hline \multirow[t]{2}{*}{$\operatorname{lnLp}(a)$} & $2.30 \pm 1.19$ & $2.43 \pm 1.03$ & $2.65 \pm 1.06$ & $P=0.428$ & $2.31 \pm 0.77$ & $2.82 \pm 0.88$ & $3.22 \pm 1.001$ & $P=0.422$ & $2.66 \pm 0.82$ & $2.76 \pm 0.92$ & $2.82 \pm 1.02$ & $P=0.841$ \\
\hline & & & & 1.259 & & & & 17.70 & & & & 0.284 \\
\hline Lp(a)(mg/dl) & 10.02 & 11.43 & 14.18 & - & 10.13 & 16.81 & 25.05 & - & 14.33 & 15.78 & 16.81 & - \\
\hline
\end{tabular}


Table 4 Genotypic lipid levels (Mean \pm SD) of the -629 C/A promoter polymorphism in the three ethnic populations for controls

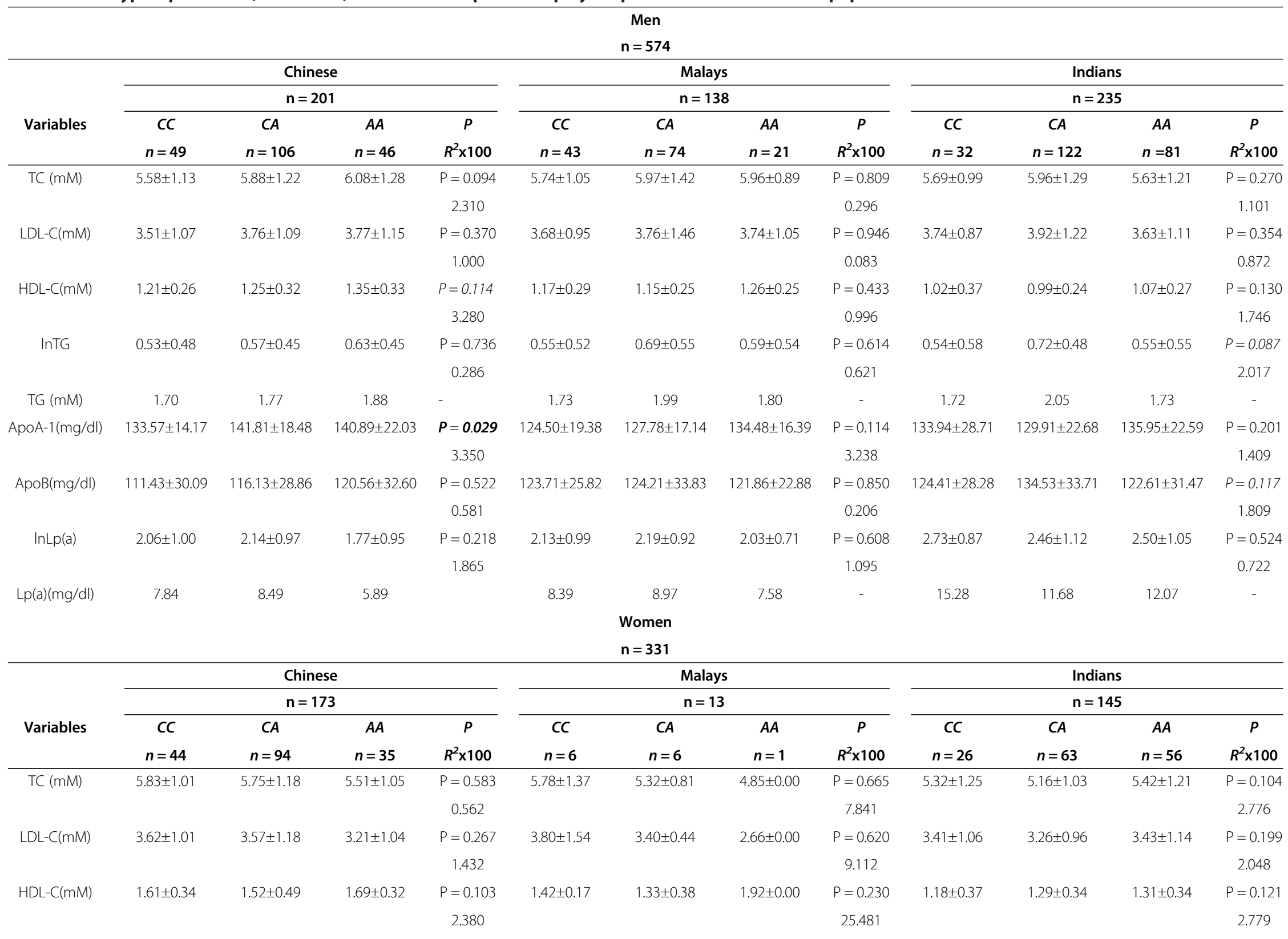


Table 4 Genotypic lipid levels (Mean \pm SD) of the -629 C/A promoter polymorphism in the three ethnic populations for controls (Continued)

\begin{tabular}{|c|c|c|c|c|c|c|c|c|c|c|c|c|}
\hline \multirow[t]{2}{*}{$\ln T G$} & $0.16 \pm 0.50$ & $0.22 \pm 0.55$ & $0.19 \pm 0.49$ & $P=0.709$ & $0.05 \pm 0.60$ & $0.11 \pm 0.57$ & $-0.54 \pm 0.00$ & $P=0.604$ & $0.20 \pm 0.69$ & $0.18 \pm 0.50$ & $0.26 \pm 0.48$ & $P=0.629$ \\
\hline & & & & 0.323 & & & & 9.592 & & & & 0.536 \\
\hline TG (mM) & 1.17 & 1.25 & 1.21 & & 1.05 & 1.12 & 0.58 & & 1.22 & 1.20 & 1.30 & \\
\hline \multirow[t]{2}{*}{ ApoA-1 (mg/dl) } & $151.86 \pm 23.78$ & $150.69 \pm 24.82$ & $148.72 \pm 21.16$ & $P=0.853$ & $146.00 \pm 11.12$ & $145.50 \pm 22.35$ & $160.00 \pm 0.00$ & $P=0.230$ & $134.44 \pm 28.66$ & $139.32 \pm 24.63$ & $147.57 \pm 27.20$ & $P=0.094$ \\
\hline & & & & 0.196 & & & & 21.127 & & & & 3.314 \\
\hline \multirow[t]{2}{*}{ ApoB(mg/dl) } & $97.29 \pm 28.20$ & $95.84 \pm 30.02$ & $88.88 \pm 26.80$ & $P=0.520$ & $100.33 \pm 23.96$ & $102.50 \pm 10.05$ & $85.00 \pm 0.00$ & $P=0.687$ & $116.48 \pm 33.29$ & $106.74 \pm 27.52$ & $117.37 \pm 34.39$ & $P=0.014$ \\
\hline & & & & 0.689 & & & & 7.222 & & & & 4.429 \\
\hline \multirow[t]{2}{*}{$\ln L p(a)$} & $2.53 \pm 1.23$ & $2.41 \pm 1.02$ & $2.45 \pm 1.07$ & $P=0.882$ & $2.77 \pm 1.01$ & $2.97 \pm 1.06$ & $2.48 \pm 0.00$ & $P=0.909$ & $2.90 \pm 1.02$ & $2.75 \pm 0.98$ & $3.00 \pm 0.89$ & $P=0.590$ \\
\hline & & & & 0.185 & & & & 2.362 & & & & 1.325 \\
\hline Lp(a)(mg/dl) & 12.60 & 11.18 & 11.55 & - & 15.95 & 19.49 & 11.94 & - & 18.11 & 15.61 & 20.12 & - \\
\hline
\end{tabular}




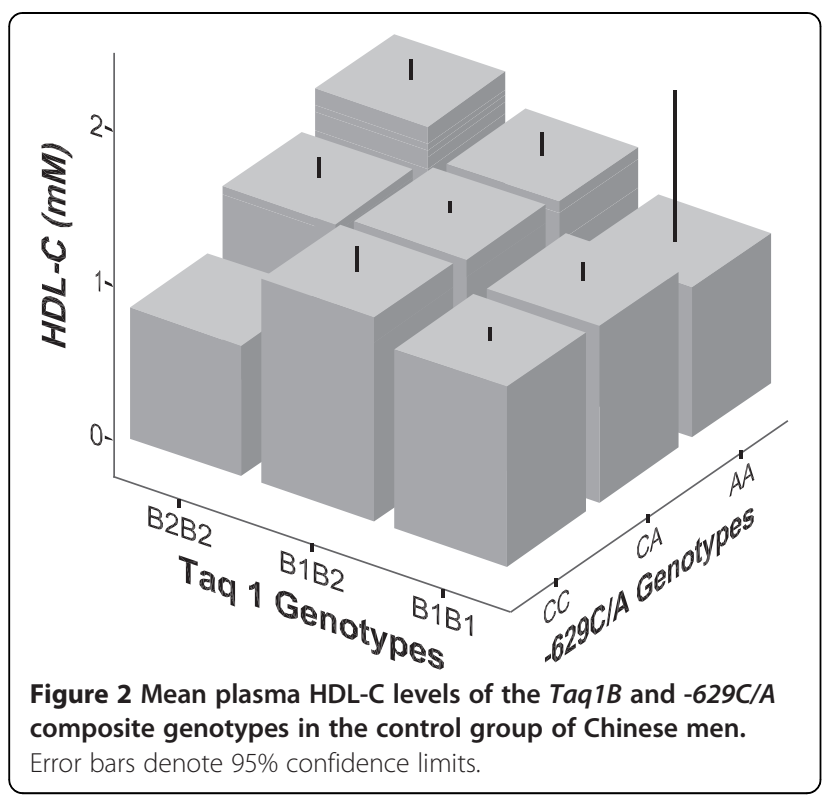

Chinese men (Figure 3C). This was also confirmed by ANCOVA performed separately for non-smokers (0.001) and smokers $(\mathrm{p}=0.943)$. One should not interpret from this figure that B1B1 smokers have higher HDL-C levels as the difference in HDL-C levels between the smokers and non-smokers is not statistically significant. A similar trend was also observed for the $-629 \mathrm{C}>\mathrm{A}$ polymorphism (Figure 3D).

\section{Discussion}

In this study we genotyped three Asian ethnic populations in Singapore for two polymorphisms of the CETP gene to determine their allele frequencies and association with $\mathrm{CAD}$ and plasma lipid levels. The allele frequencies of the Taq1B polymorphism showed marked ethnic differences. The $B 1$ allele is most common in the Malays but is the minor allele in the Indians. The minor B2 allele in the Chinese and Malays are close to most Caucasian frequencies reported [38].The allele frequencies of Taq1B polymorphism in Indians, Malays and Chinese are consistent with the respective studies conducted previously in Sri Lankans [25], Taiwanese [27] and Singaporeans [33]. Similar to B1, the $-629 C$ allele is also the most common in the Malays $(0.60)$ but is the minor allele in the Indians (0.30).

Among the three major ethnic groups in Singapore, the Chinese is the only group that shows a significantly lower frequency of the $B 2$ allele in CAD + cases compared to the controls. This finding is consistent with observation in the Caucasians [11] and suggests the association of the $B 2$ with some protective factors of CAD in Chinese population. Chinese subjects without the $B 2$ allele have 2.0 times higher risk of CAD relative to those who have at least one copy of the $B 2$ allele. This is independent of the effects of age, smoking, BMI, gender, hypertension, dyslipidemia and diabetes mellitus. However, this effect is not observed in the Malays and Indians. In the Taiwanese, the odds ratio for CAD was slightly higher for the $B 1 B 1$ than $B 2 B 2$ group, but no significant difference in Taq1B allele distribution was observed between the control and CAD groups [27]. Padmaja et al. [31] demonstrated that CETP $B 1 B 1$ of Taq1B was significantly associated with increased risk for CAD (OR 2.7; 95\% CI 1.5-3.3) in the Indian population. The $B 2$ allele is unlikely to be a functional mutation as its position in the intron is not known to affect RNA splicing or serves any other regulatory purposes. As such, any phenotype that it is associated has to be due to its LD with another functional site that is either within the CETP gene or its functionally related genes nearby, such as the lecithin-cholesterol acetyl transferase gene. Dachet et al. [22] first reported the strong LD between $-629 C>A$ and Taq1B polymorphisms. Later many more alleles in the CETP gene [39-41] were also reported to be in LD with the Taq1B site. In the Singaporean population, weak LD between Taq1B and $-629 C>A$ was consistently observed in all three ethnic groups, although to varying extent. It is highest in the Malays, followed by Chinese and Indians. Wu et al. [23] observed a LD between Taq1B and -629C > A polymorphic sites in the Chinese population from China.

The effect of the Taq1B intronic polymorphism in this study could not be generalized across ethnic groups and genders. This is expected since the magnitude of its effects is dependent upon the strength of its LD with a functional site. Among the lipid traits that we have studied, the $B 2$ allele is most evidently associated with raised levels of protective factors such as HDL-C and its associated apoA1. This trend of association was consistent in all ethnic groups and genders although statistical significance was attained only in the Chinese men. Significant association of the $B 2$ allele with elevated HDL-C was also reported for the Framingham population [11], Chinese population [23], Iranian population $[19,20]$ and Tunisian population [9]. In their studies, the protective effects of the $B 2$ allele on the development of CAD were observed in association with increased HDL-C and decreased CETP activity. The association of the $B 2$ allele with higher plasma HDL-C and / or apoA1 has been consistently observed in many other studies $[15,24,27,28,33,42]$ as well. However, Rahimi et al. [14] indicated that the CETPB1 allele is associated with increased risk of CAD and type 2 diabetes mellitus independent of plasma HDL-C level in the Iranian population.

In this study, we have shown that the association of the Taq1B site with HDL-C and apoA1 levels remains independent of the effects of the promoter $-629 C>A$. Both sites have similar effects on apoA1. We asked the question of whether the $-629 C>A$ site is the functional mutation while Taq1B was merely showing significant 

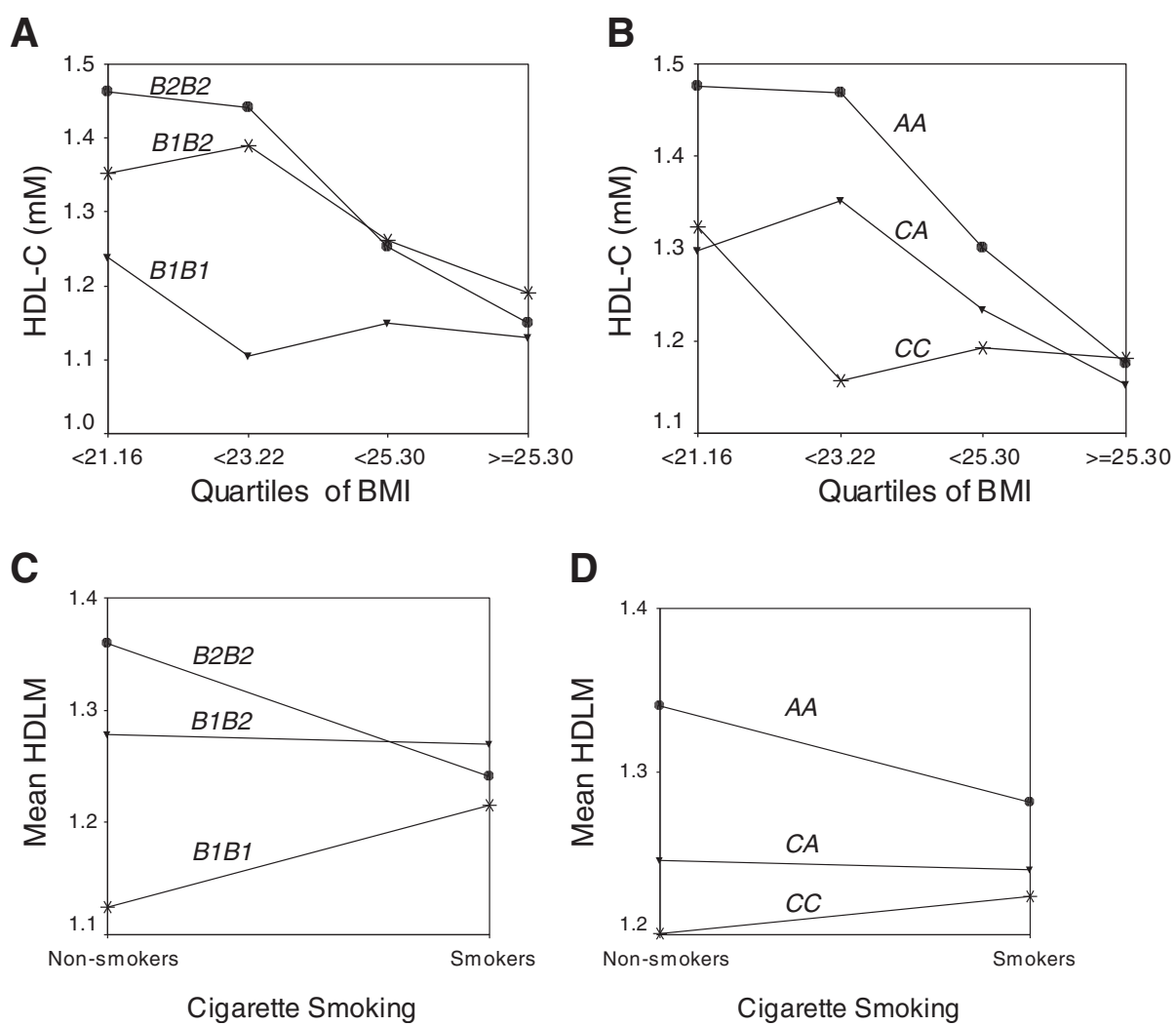

Figure 3 Mean genotypic plasma HDL-C levels in the control group of Chinese men according to A) BMI quartiles and Taq1B genotypes; B) BMI quartiles and -629C > A genotypes; C) Cigarette smoking and Taq1B genotypes; D) Cigarette smoking and -629C $>A$ genotypes.

association with plasma lipid levels as a result of LD. We concluded that this may not be the case. Firstly, a functional mutation is likely to have its effect observed across all ethnic groups and genders. However, we observed effects of the promoter polymorphism only in Chinese men. Although we cannot be conclusive that the $-629 C>A$ polymorphism is not a functional mutation since other genes and environmental factors could have masked its moderate effect, its ethnic-and genderspecific effects nevertheless suggests the unlikelihood of this being so. Secondly, the strength of association with HDL-C and apoA1 levels was consistently higher for the Taq1B site than the promoter site. This should not be the case if the $-629 C>A$ site is functional and Taq1B site its marker. Moreover, in the two-way ANCOVA, there was no effect of the promoter polymorphism when the Taq1B site was included in the model. We postulated that there could be other functional sites within or near the CETP gene that the Taq1B site is in stronger LD with than the promoter site. Both Taq1B and $-629 \mathrm{C}>\mathrm{A}$ polymorphisms could possibly have independent effects on HDL-C and apoA1 levels and hence they do not show significant effects individually when they are included in the same 2-way ANCOVA model. In the Turkish population, it was found that CETP $-629 C>A$ polymorphism was not associated with CAD [43]. As with any complex traits, the genotypic effect is usually confounded by environmental factors. In this study, we found significant geneenvironmental interaction of the Taq1B polymorphism with BMI and smoking. The protective effect of the $B 2$ allele was only observed in subjects having BMI $<23$ and in non-smokers. This is consistent with other studies that examined the confounding effects of BMI $[21,28,38]$ and smoking $[15,25,38,44]$.

We concluded that, i) the absence of the $B 2$ allele was associated with CAD in the Chinese, ii) the minor alleles of the two polymorphisms were significantly associated with higher plasma HDL-C levels in the Chinese men (B2), iii) the effects of the polymorphisms were significant only in non-smoking subjects with BMI up to the $50^{\text {th }}$ percentile, iv) The effect of the Taq1B polymorphism is not entirely dependent on the $-629 \mathrm{C} / \mathrm{A}$ site but that it could be in LD with some other functional sites.

Competing interests

The authors declare that they have no competing interests. 


\section{Authors' contributions}

YL carried out genotyping. NT and CKH carried out data analysis. HL assisted in the genotyping. NS was responsible for subject recruitment. $\mathrm{HY}$ and $\mathrm{CKH}$ contributed to the conception and design of this study. NT and $\mathrm{CKH}$ revised the manuscript. All authors read and approved the final manuscript.

\section{Acknowledgements}

This study was supported in part by the National Medical Research Council, Singapore (Grant NMRC/1155/2008, NMRC/0408/2000) and the Agency for Science, Technology and Research, Singapore (Grant NSTB EMT/00/022/ (ADRA). The authors gratefully acknowledge the excellent editorial assistance from Dr Dimple Rajgor.

\section{Author details}

'Department of Paediatrics, Yong Loo Lin School of Medicine, National University of Singapore, Singapore, Singapore. ${ }^{2}$ Department of Biochemistry, National University of Singapore, Singapore, Singapore. ${ }^{3}$ School of Biotechnology and Biomolecular Sciences, The University of New South Wales, Sydney 2052, Australia.

Received: 13 December 2012 Accepted: 24 May 2013

Published: 8 June 2013

\section{References}

1. Miller GJ, Miller NE: Plasma-high-density-lipoprotein concentration and development of ischaemic heart-disease. Lancet 1975, 1:16-19.

2. Robins SJ, Collins D, Wittes JT, et al: Veterans Affairs High-Density Lipoprotein Intervention Trial. Relation of gemfibrozil treatment and lipid levels with major coronary events: VA-HIT: a randomized controlled trial. JAMA 2001, 28(285):1585-1591.

3. Borggreve $S E$, De Vries R, Dullaart RP: Alterations in high-density lipoprotein metabolism and reverse cholesterol transport in insulin resistance and type 2 diabetes mellitus: role of lipolytic enzymes, lecithin: cholesterol acyl transferase and lipid transfer proteins. Eur J Clin Invest 2003, 33:1051-1069.

4. Schwartz CC, Vanden Broek JM, Cooper PS: Lipoprotein cholesteryl ester production, transfer, and output in vivo in humans. J Lipid Res 2004, 45:1594-1607.

5. Fielding CJ, Fielding PE: Molecular physiology of reverse cholesterol transport. J Lipid Res 1995, 36:211-228.

6. Barter PJ, Rye KA: Cholesteryl ester transfer protein, high density lipoprotein and arterial disease. CurrOpinLipidol 2001, 12:377-382.

7. Yamashita S, Hirano K, Sakai N, et al: Molecular biology and patholophysiological aspects of plasma cholesteryl ester transfer protein. Biochim Biophys Acta 2000, 1529:257-275.

8. Thompson A, Di Angelantonio E, Sarwar N, et al: Association of cholesteryl ester transfer protein genotypes with CETP mass and activity, lipid levels, and coronary risk. JAMA 2008, 18(299):2777-2788.

9. Rejeb J, Omezzine A, Boumaiza I, et al: Four polymorphisms of cholesteryl ester transfer protein gene and coronary stenosis in a Tunisian population. J Cardiovasc Med (Hagerstown) 2012, 13:546-553.

10. Kuivenhoven JA, Jukema JW, Zwinderman AH, et al: The role of a common variant of the cholesteryl ester transfer protein gene in the progression of coronary atherosclerosis. The Regression Growth Evaluation Statin Study Group. N Engl J Med 1998, 338:86-93.

11. Ordovas JM, Cupples LA, Corella D, et al: Association of cholesteryl ester transfer protein-TaqIB polymorphism with variations in lipoprotein subclasses and coronary heart disease risk. Arterioscler Thromb Vasc Biol 2000, 20:1323-1329.

12. Arca M, Montali A, Ombres D, et al: Lack of association of the common TaqIB polymorphism in the cholesteryl ester transfer protein gene with angiographically assessed coronary atherosclerosis. Clin Genet 2001, 60(5):374-380

13. Cuchel M, Wolfe ML, deLemos AS, et al: The frequency of the cholesteryl ester transfer protein-Taql B2 allele is lower in African Americans than in Caucasians. Atherosclerosis 2002, 163(1):169-174.

14. Rahimi Z, Nourozi-Rad R, Vaisi-Raygani A, et al: Association between cholesteryl ester transfer protein TaqIB variants and risk of coronary artery disease and diabetes mellitus in the population of western Iran. Genet Test Mol Biomarkers 2011, 15:813-819.
15. Kondo I, Berg K, Drayna D, et al: DNA polymorphism at the locus for human cholesteryl ester transfer protein (CETP) is associated with high density lipoprotein cholesterol and apolipoprotein cholesterol and apolipoprotein levels. Clin Genet 1989, 35:49-56.

16. Freeman DJ, Packard CJ, Shepherd J, et al: Polymorphisms in the gene coding for cholesteryl ester transfer protein are related to plasma highdensity lipoprotein cholesterol and transfer protein activity. Clin Sci 1990, 79:575-581.

17. Gudnason V, Kakko S, Nicaud V, et al: Cholesteryl ester transfer protein gene effect on CETP activity and plasma high-density lipoprotein in European populations. Eur J Clin Invest 1999, 29:116-128.

18. Corella D, Saiz C, Guillen M, et al: Association of TaqIB polymorphism in the cholesteryl ester transfer protein gene with plasma lipid levels in a healthy Spanish population. Arteriosclerosis 2000, 152:367-376.

19. Hassanzadeh T, Firoozrai M, Zonouz AE, et al: Taq1B polymorphism of cholesteryl ester transfer protein (CETP) gene in primary combined hyperlipidaemia. Indian J Med Res 2009, 129:293-298.

20. Kashani Farid MA, Azizi F, Hedayati M, et al: Association between CETP Taq1B and LIPC -514C/T polymorphisms with the serum lipid levels in a group of Tehran's population: a cross sectional study. Lipids Health Dis 2010, 7:9-96.

21. Ruan X, Ma L, Wang S, et al: Association of two CETP polymorphisms with HDL levels in the Chinese obese population. Obesity (Silver Spring) 2009, 17:2196-2201

22. Dachet C, Poirier O, Cambien F, Chapman J, Rouis M: New functional promoter polymorphism, CETP/-629, in cholesteryl ester transfer protein (CETP) gene related to CETP mass and high density lipoprotein cholesterol levels: role of Sp1/Sp3 in transcriptional regulation. Arterioscler Thromb Vasc Biol 2000, 20(2):507-515.

23. Wu Y, Bai H, Liu R, et al: Analysis of cholesterol ester transfer protein gene Taq IB and -629 C/A polymorphisms in patients with endogenous hypertriglyceridemia in Chinese population. Zhonghua Yi Xue Yi Chuan XueZaZhi 2006, 23:640-646.

24. Eiriksdottir G, Bolla MK, Thorsson B, et al: The $-629 \mathrm{C}>$ A polymorphism in the CETP gene does not explain the association of TAQIB polymorphism with risk and age of myocardial infarction in Icelandic men. Atherosclerosis 2001, 159:187-192

25. Mendis S, Shepherd J, Packard CJ, et al: Genetic variation in the cholesteryl ester transfer protein and apoliprotein A-I genes and its relation to coronary heart disease in a Sri Lanka population. Atherosclerosis 1990, 83:21-27.

26. Song GJ, Han GH, Chae JJ, et al: The effects of the cholesterol ester transfer protein gene and environmental factors on the plasma high density lipoprotein cholesterol levels in the Korean population. Mol Cells 1997, 7:615-619.

27. $\mathrm{Wu} \mathrm{JH}$, Lee YT, Hsu HC, et al: Influence of CETP gene variation on plasma lipid levels and coronary heart disease: a survey in Taiwan. Atherosclerosis 2001, 159:451-458.

28. Hsu LA, Ko YL, Hsu KH, et al: Genetic variations in the cholesteryl ester transfer protein gene and high density lipoprotein cholesterol levels in Taiwanese Chinese. Hum Genet 2002, 110:57-63.

29. Zheng K, Zhang S, Zhang L, et al: Carriers of three polymorphisms of cholesteryl ester transfer protein gene are at increased risk to coronary heart disease in a Chinese population. Int J Cardiol 2005, 103(3):259-265.

30. Padmaja N, Ravindra Kumar M, Soya SS, et al: Common variants of Cholesterylestertransfer protein gene and their association with lipid parameters in healthy volunteers of Tamilian population. Clin Chim Acta 2007, 375:140-146.

31. Padmaja N, Kumar RM, Balachander J, et al: Cholesteryl ester transfer protein TaqIB, $-629 \mathrm{C}>\mathrm{A}$ and $1405 \mathrm{~V}$ polymorphisms and risk of coronary heart disease in an Indian population. Clin Chim Acta 2009, 402:139-145.

32. Yilmaz H, Isbir T, Agachan B, et al: Effects of cholesterol ester transfer proteinTaq1B gene polymorphism on serum lipoprotein levels in Turkish coronary artery disease patients. Cell Biochem Funct 2005, 23:23-28.

33. Tai ES, Ordovas JM, Corella D, et al: The Taq1B and $-629 C>A$ polymorphisms at the cholesteryl ester transfer protein locus: associations with lipid levels in a multiethnic population. The 1998 Singapore National Health Survey. Clin Genet 2003, 63(1):19-30.

34. Hughes K, Lun KC, Yeo PP: Cardiovascular diseases in Chinese, Malays, and Indians in Singapore. I. Differences in mortality. J Epidemiol Community Health 1990, 44:24-28. 
35. Saha N: Serum high density lipoprotein cholesterol, apolipoprotein A-I, A-II and B levels in Singapore ethnic groups. Atherosclerosis 1987, 68:117-121.

36. Heng CK, Saha N, Tay JS: Lack of association of apolipoprotein E polymorphism with plasma Lp(a) levels in the Chinese. Clin Genet 1995, 48:113-119

37. VanLiere JM, Rosenberg NA: Mathematical properties of the $r^{2}$ measure of linkage Disequilibrium. Theor Popul Biol 2008, 74:130-137.

38. Freeman DJ, Griffin BA, Holmes AP, et al: Regulation of Plasma HDL Cholesterol and Subfraction Distribution by Genetic and Environmental Factors. Associations between the Taql B RFLP in the CETP gene and smoking and obesity. ArteriosclerThromb 1994, 14:336-344.

39. Corbex $\mathrm{M}$, Poirier $\mathrm{O}$, Fumeron $\mathrm{F}$, et al: Extensive association analysis between the CETP gene and coronary heart disease phenotypes reveals several putative functional polymorphisms and gene-environment interaction. Genet Epidemiol 2000, 19:64-80.

40. Kakko S, Tamminen M, Paivansalo M, et al: Variation at the cholestery ester transfer protein gene in relation to plasma high density lipoproteins cholesterol levels and carotid intima-media thickness. Eur $\int$ Clin Invest 2001, 31:593-602.

41. Goff WL, Guerin M, Nicaud V, et al: A novel cholesteryl ester transfer protein promoter polymorphism (-917G/A) associated with plasma high-density lipoprotein cholesterol levels. Interaction with the Taq1B and -629 C > A polymorphisms. Atherosclerosis 2002, 161:269-279.

42. Fumeron F, Betoulle $D$, Luc G, et al: Alcohol intake modulates the effect of a polymorphism of the cholesteryl ester transfer protein gene on plasma high density lipoprotein and the risk of myocardial infarction. J Clin Invest 1995, 96:1664-1671.

43. Tanrikulu S, Ademoglu E, Gurdol F, et al: Association of cholesteryl ester transfer protein $-629 \mathrm{C}>\mathrm{A}$ polymorphism with high-density lipoprotein cholesterol levels in coronary artery disease patients. Cell Biochem Funct 2009, 27:452-457.

44. Hannuksela ML, Liinamaa MJ, Kesaniemi YA, et al: Relation of polymorphisms in the cholesteryl ester transfer protein gene to transfer protein activity and plasma lipoprotein levels in alcohol drinkers. Atherosclerosis 1994, 110:35-44.

doi:10.1186/1476-511X-12-85

Cite this article as: Lu et al:: Association of CETP Taq1B and -629 C > A polymorphisms with coronary artery disease and lipid levels in the multi-ethnic Singaporean population. Lipids in Health and Disease 2013 12:85.

\section{Submit your next manuscript to BioMed Central and take full advantage of:}

- Convenient online submission

- Thorough peer review

- No space constraints or color figure charges

- Immediate publication on acceptance

- Inclusion in PubMed, CAS, Scopus and Google Scholar

- Research which is freely available for redistribution 